\title{
Estilos de aprendizaje en los estudiantes de enfermería
}

\author{
Learning styles in nursing students
} Ma del Coro Canalejas Pérez, M ${ }^{\mathrm{a}}$ Luisa Martínez Martín, Ma Cristina Pineda Ginés, Manuel Luis Vera Cortés,
Marina Soto González, Ángela Martín Marino, Ma Luisa Cid Galán.

Escuela Universitaria de Enfermería La Paz.

Introducción: El objetivo de este trabajo es identificar el estilo de aprendizaje predominante en los estudiantes de enfermería de nuestro centro, como punto de partida para una reflexión sobre el proceso de aprendizaje-enseñanza que estamos llevando a cabo.

Material y métodos: Se realizó un estudio observacional, exploratorio y transversal en la Escuela de Enfermería La Paz durante el curso académico 2002-03 con una muestra de 180 estudiantes. Las variables seleccionadas fueron: estilo de aprendizaje, curso en el que estaban matriculados, vía de acceso a los estudios y experiencia laboral. El instrumento que se utilizó fue el cuestionario de estilos de aprendizaje Honey-Alonso (CHAEA). El análisis de los datos consistió en el contraste de las diferencias entre las puntuaciones obtenidas en los cuatro estilos de aprendizaje, mediante la prueba ANOVA y el test de Kruskal-Wallis.

Resultados: Se encontraron diferencias estadísticamente significativas en los estilos de aprendizaje reflexivo y teórico, en función del año de carrera que estaban cursando. No se encontraron diferencias estadísticamente significativas entre los cursos para los estilos de aprendizaje activo y pragmático.
Discusión: El perfil de aprendizaje de los estudiantes de nuestro centro es diferente entre los alumnos de los tres cursos, existiendo una tendencia hacia un estilo más reflexivo y teórico, a medida que avanzan en su formación.

Es necesario buscar alternativas para potenciar el desarrollo de los estilos activo y pragmático en la misma medida que los estilos reflexivo y teórico

Palabras clave: Estilo de aprendizaje, estudiante de enfermería, cuestionario CHAEA, enseñanza en Enfermería.

Introduction: This paper aims to identify the prevalent learning style among nursing students at our teaching institution, as a starting point for an analysis of the current learning-teaching process.

Methods and materials: An observational, exploratory and cross-sectional study was performed in the Nursing School of La Paz (Universidad Autónoma de Madrid), during the academic year 2002-2003, in a sample comprising 180 students. The variables selected were: learning style, academic year, form of access to nursing studies and previous work experience. The instrument used was the Honey-Alonso (CHAEA) learning style questionnaire. Data analysis was based on the contrasting of the differences between the scores in the four learning styles, through the ANOVA test and the Kruskal-Wallis test.

Results: Statistically significant differences were found in the reflexive and theoretic learning styles, and no significant differences were found for the active and pragmatic learning styles, according to the students' academic year.

Discussion: Learning styles in our teaching institution evolve as students progress through their studies. There is an increasing tendency towards the reflexive
$\mathrm{M}^{\mathrm{a}}$ del Coro Canalejas Pérez

Escuela Universitaria de Enfermería La Paz

Paseo de la Castellana 261

28046 Madrid

Teléfono y fax: 917277136

Correo electrónico: ccanalejas.hulp@salud.madrid.org 
and theoretic styles at upper levels. Alternatives should be explored in order to develop the active and pragmatic learning styles.

Key words: Learning styles, nursing students, CHAEA questionnaire, nursing teaching.

\section{INTRODUCCIÓN}

La sociedad actual demanda personas que, a través de un aprendizaje continuo, se conviertan en sujetos autónomos, capaces de tomar conciencia de sus propios procesos mentales al enfrentarse con los problemas, analizarlos adecuadamente, planificar, supervisar y evaluar la propia actuación ${ }^{1}$.

Las investigaciones cognitivas han demostrado que las personas aprenden de diversas formas ${ }^{2}$, lo que hace necesaria la reflexión sobre las variables que influyen en la capacidad de aprender. Una de estas variables es el estilo de aprendizaje o tendencia a desarrollar unas preferencias globales en la elección de estrategias para aprender ${ }^{3}$. La comprensión del propio estilo por parte del estudiante es uno de los factores claves para desarrollar la capacidad de aprender a aprender.

Los estilos de aprendizaje $\mathrm{e}^{4,5}$, aunque son relativamente estables, pueden ser modificados, siendo una responsabilidad de los docentes ayudar a los estudiantes a descubrir su estilo y aprender a adaptarlo a las experiencias de cada situación.

Entre los estudios sobre estilos de aprendizaje, destacan los que van encaminados a la validación de instrumentos que facilitan el diagnóstico de los mismos y, por lo tanto, el autoconocimiento del discente. Entre ellos podemos señalar a Kolb, Honey, Munford y Alonso, que han centrado sus investigaciones en el aprendizaje experiencial y en la influencia de los estilos en el mismo. Estos autores consideran que el proceso de aprender implica un recorrido cíclico por cuatro etapas sucesivas: tener una experiencia, reflexionar sobre ella, extraer conclusiones y planificar los pasos siguientes a aplicar. Cada discente recorre las cuatro etapas, aunque muestra preferencias distintas por cada una de ellas, lo que define su estilo de aprendizaje. Honey y Munford, y posteriormente Alonso, denominaron los estilos de aprendizaje relacionados con cada una de las etapas del ciclo de la siguiente forma ${ }^{7}$ :

- Estilo activo: Indica una preferencia por impli- carse en nuevas experiencias e involucrarse plenamente en el trabajo de equipo. Suelen ser personas animadoras, improvisadoras, descubridoras, arriesgadas y espontáneas.

- Estilo reflexivo: Prefieren observar la experiencia desde diferentes perspectivas. Recogen datos y los analizan detenidamente, son ponderados, concienzudos, receptivos, analíticos y exhaustivos.

- Estilo teórico: Les gusta adaptar e integrar las observaciones dentro de teorías lógicas y complejas. Son profundos en su sistema de pensamiento, metódicos, lógicos, objetivos, críticos y estructurados.

- Estilo pragmático: Prefieren la aplicación práctica de las ideas. Son experimentadores, prácticos, directos, eficaces y realistas.

Desde 1980, los investigadores de enfermería han mostrado un especial interés en el análisis de los estilos de aprendizaje en enfermeras. Autores como Highfield ${ }^{8}$; Rakoczy y Money'; Cavanagh, Hogan y Ramgopal ${ }^{10}$; y Linares $^{11}$, estudiaron los estilos de aprendizaje en estudiantes de enfermería utilizando el cuestionario de Kolb ${ }^{12}$.

En nuestro país, el primer estudio realizado sobre estilos de aprendizaje en alumnos de enfermería, es la tesis doctoral de Alonso ${ }^{13}$ sobre estilos de aprendizaje en estudiantes universitarios. Entre los centros universitarios que seleccionó se encontraba una escuela de enfermería con una muestra de 89 estudiantes de 3 er curso. El instrumento utilizado fue el cuestionario de estilos de aprendizaje Honey-Alonso $(\mathrm{CHAEA})^{13}$, que fue validado en dicho estudio. Entre las conclusiones destacaban que el perfil de aprendizaje de los estudiantes de enfermería es de preferencia muy alta para el estilo reflexivo y muy baja para el estilo activo.

En los años que han transcurrido desde la investigación de Alonso, se han producido cambios significativos en la formación de enfermeras y en el desarrollo de la propia disciplina. En este sentido, parece necesario analizar de nuevo el perfil de aprendizaje de los estudiantes de enfermería al considerarlo un indicador útil para conocer el modo en que se están orientando sus preferencias por aprender de diversas formas.

Con este trabajo se pretendía conocer el estilo de aprendizaje de los estudiantes de enfermería y analizar su relación con el curso que están realizando.

\section{MATERIAL Y MÉTODOS}

Se realizó un estudio observacional, exploratorio y transversal, durante el curso académico 2002-03, 
en la Escuela de Enfermería La Paz, adscrita a la Universidad Autónoma de Madrid.

La muestra la constituyeron 180 estudiantes matriculados en los tres cursos, que participaron de forma voluntaria.

Las variables seleccionadas fueron:

- Variable principal: Estilo de aprendizaje.

- Variables secundarias: Curso en el que estaban matriculados, vía de acceso a los estudios de Enfermería y experiencia laboral.

El instrumento utilizado para medir las variables fue el Cuestionario Honey-Alonso de Estilos de Aprendizaje (CHAEA) ${ }^{13}$, al que se le incorporó una ficha para la recogida de datos relacionados con las variables secundarias (anexo). Dicho cuestionario consta de 80 items de respuesta dicotómica, de los cuales 20 corresponden a cada estilo de aprendizaje y están distribuidos aleatoriamente. La puntuación absoluta que cada sujeto obtiene en cada grupo de 20 items, indica el nivel que alcanza en cada uno de los cuatro estilos. De esta forma se obtienen los datos precisos para valorar las preferencias de cada alumno en cada uno de los estilos y obtener, por tanto, su perfil de aprendizaje.

El trabajo de campo se realizó en diferentes periodos del año académico, en función del curso que los estudiantes estaban realizando. En este sentido, para los alumnos de primero se eligió el comienzo del curso con el fin de identificar el perfil de estilo de aprendizaje con el que llegaban al centro; para segundo se seleccionó el mes de febrero con el objetivo de valorar posibles cambios de estilo a la mitad de la carrera, y finalmente para tercer curso se eligió el mes de abril cercano a la finalización de los estudios de Enfermería.

Para procesar la información de los cuestionarios se elaboró una base de datos en el programa Excel y se realizó un análisis estadístico con el programa SPSS versión 13.3. Dicho análisis consistió en:

- Descripción de la muestra.

- Comparación cualitativa de las puntuaciones medias obtenidas en los cuatro estilos de aprendizaje en los tres cursos.

- Contraste de las diferencias entre las puntuaciones obtenidas en los cuatro estilos de aprendizaje en los tres cursos. Se aplicó la prueba ANOVA para los estilos reflexivo y teórico y las pruebas de contraste de Scheffe y Bonferroni, teniendo en cuenta la homogeneidad de las varianzas. Así mismo se aplicó el test de Kruskal-Wallis para los estilos activo y pragmático, dado que existían diferencias significativas en las varianzas.
- Comparación, a través de la prueba t de Student, de las medias obtenidas en los cuatro estilos de aprendizaje y las variables vía de acceso a los estudios de Enfermería y experiencia laboral previa de los estudiantes.

\section{RESULTADOS}

\section{Descripción de la muestra}

El número total de estudiantes que cumplimentó el cuestionario fue de 180 (90\%) de un total de 200 alumnos matriculados. De estos correspondían 75 $(41,7 \%)$ a primer curso, $60(33,3 \%)$ a segundo y 45 $(25 \%)$ a tercero.

La mayoría de los participantes en el estudio eran mujeres $(91,7 \%)$, manteniéndose porcentajes similares en los tres cursos. La edad media fue de 20,23 años (DE: 2,53).

El $63,3 \%$ de los estudiantes manifestó no tener experiencia laboral, frente a un $36,7 \%$ que sí la tenía, principalmente en actividades sin relación con el campo de la salud.

La principal vía de acceso a los estudios de enfermería fue la selectividad $(82,8 \%)$, frente a otras formas de ingreso como la vía de formación profesional $(11,1 \%)$ o la opción de mayores de 25 años $(6,1 \%)$.

\section{Análisis descriptivo y comparativo de los estilos de aprendizaje por curso}

El perfil de aprendizaje de los estudiantes de nuestro centro mostró un predominio de los estilos reflexivo y teórico (fig 1.). Al comparar los perfiles de

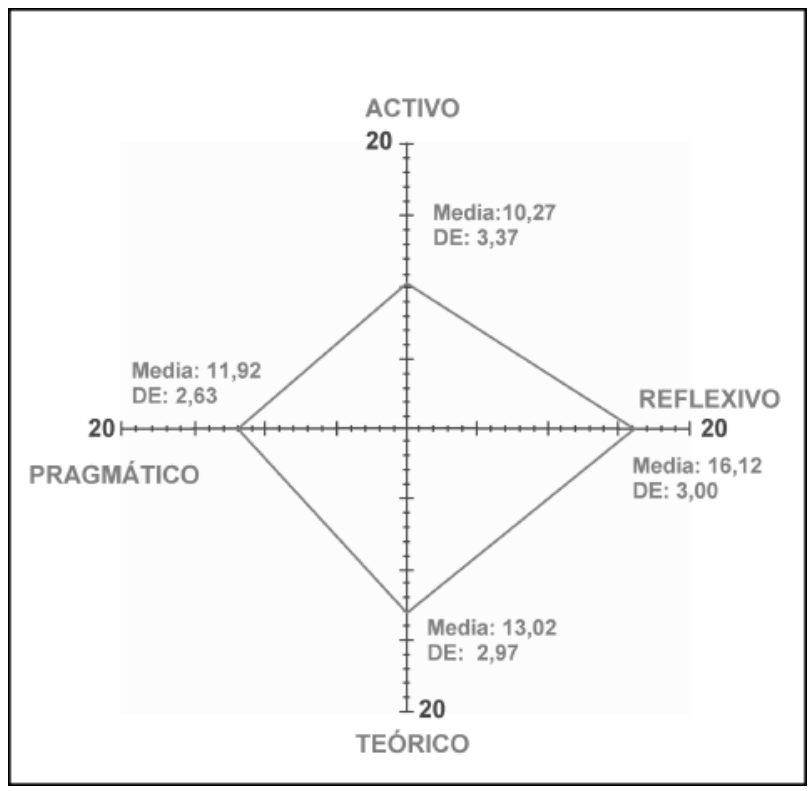

Fig.1 Perfil de aprendizaje de la muestra 


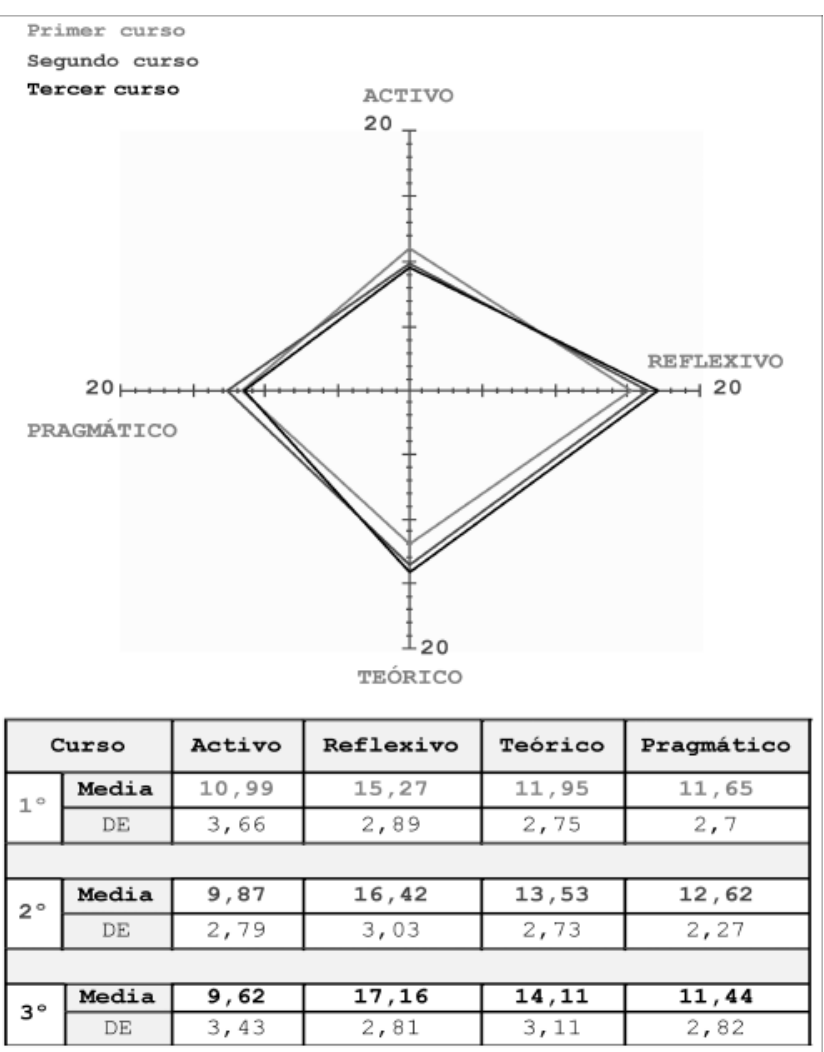

Fig.2 Comparación del perfil de aprendizaje de los alumnos de los tres cursos

aprendizaje de los tres cursos, se observó que en el estilo activo las puntuaciones iban descendiendo a medida que avanzan los cursos; mientras que en los estilos reflexivo y teórico sucedía lo contrario (fig.2). Finalmente, al comparar el perfil de los alumnos de tercer curso con el obtenido por Alonso ${ }^{13}$ en su estudio, se observó igualmente mayor preferencia por los estilos reflexivo y teórico en los estudiantes de nuestro centro (fig 3.).

\section{Contraste de las diferencias entre las puntuaciones obtenidas en los cuatro estilos en los tres cursos}

El test de homogeneidad de varianzas señaló diferencias significativas para los estilos activo (p: $0,045)$ y pragmático (p: 0,029), por esta razón se realizó la prueba ANOVA con los estilos reflexivo (p: 0,002 ) y teórico ( $\mathrm{p}: 0,000$ ) obteniéndose resultados estadísticamente significativos (Tabla 1). Con el contraste de Scheffé y Bonferroni, se obtuvo que la media de puntuación en estilo reflexivo de los alumnos de primer curso era estadísticamente inferior ( $\mathrm{p}$ : 0,002) a la obtenida por los alumnos de tercero. Así

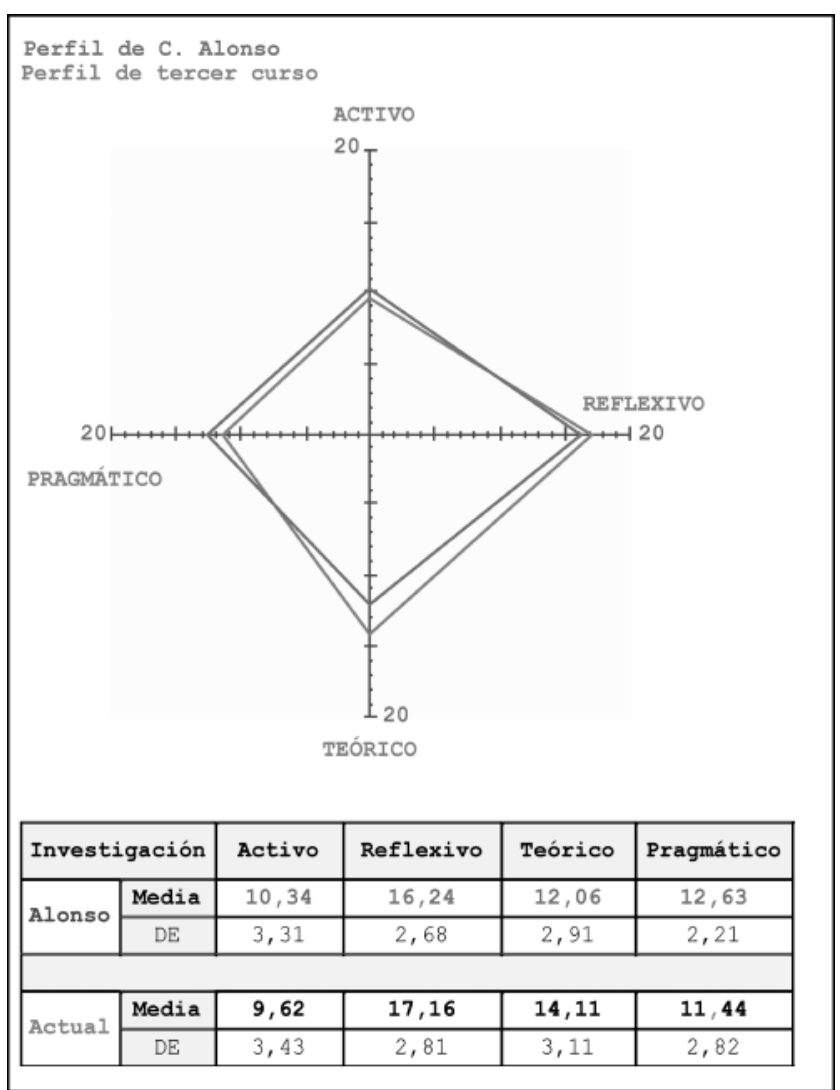

Fig.3 Comparación del estilo de aprendizaje de alumnos de tercero y estudio de Alonso

mismo, la media de puntuación en estilo teórico de los alumnos de primer curso era estadísticamente inferior a la de los alumnos de segundo (p:0,004) y tercer cursos (p: 0,000).

Para los estilos activo y pragmático, en los que no se partía de la homogeneidad de varianzas, no se obtuvieron diferencias significativas entre los cursos con el test de Kruskal-Wallis.

\begin{tabular}{|c|c|c|c|c|c|}
\hline \multicolumn{7}{|c|}{ ANOVA } \\
\hline Estilos: & $\begin{array}{c}\text { Suma de } \\
\text { cuadrados }\end{array}$ & df & $\begin{array}{c}\text { Media de } \\
\text { cuadrados }\end{array}$ & F & $\begin{array}{c}\text { Significació } \\
\text { n }\end{array}$ \\
\hline REFLEXIVO & \multicolumn{7}{|c|}{} \\
\hline Intergrupos & 108,15 & 2 & 54,07 & 6,34 & 0,002 \\
\hline Intragrupos & 1509,16 & 177 & 8,53 & & \\
\hline Total & 1617,31 & 179 & & & \\
\hline TEÓRICO & & & & \\
\hline Intergrupos & 155,79 & 2 & 77,89 & 9,66 & 0,000 \\
\hline Intragrupos & 1427,16 & 177 & 8,06 & & \\
\hline Total & 1582,95 & 179 & & & \\
\hline
\end{tabular}

Tabla 1. Contraste de las diferencias entre las puntuaciones obtenidas en estilo reflexivo y teórico en los tres cursos 


\section{Relación entre el estilo de aprendizaje y otras variables}

La aplicación del test de la t de Student para valorar la posible relación entre el estilo de aprendizaje de los estudiantes con las variables experiencia laboral y vía de acceso a los estudios de Enfermería no dio resultados estadísticamente significativos.

\section{DISCUSIÓN}

De los resultados obtenidos con esta investigación se desprende que el perfil de aprendizaje de los estudiantes de nuestro centro se caracteriza por una tendencia hacia un estilo más reflexivo y teórico, a medida que avanzan en su formación. Estos hallazgos coinciden con los obtenidos por Highfield ${ }^{8}$, Alonso $^{13}$, Rakoczy y Money ${ }^{9}$, y Linares ${ }^{11}$, llamando la atención que, a pesar del tiempo transcurrido y de ser estudios realizados en contextos tan diferentes, se hayan obtenido unos resultados similares. Es posible, como afirmaba Alonso ${ }^{13}$, que los estudiantes de enfermería tengan un estilo de aprendizaje propio $y$, por lo tanto, un "perfil de aprendizaje" en el que se diferencian claramente sus preferencias altas y bajas en la forma de aprender. Parece ser que a medida que van desarrollando competencias en el ámbito de la propia disciplina van modificando sus preferencias, de forma que van adquiriendo más interés por la observación, el análisis de los hechos y el trabajo metódico, sistematizado y lógico; y van perdiendo el interés por aprender descubriendo, experimentando, aplicando y llevando a la práctica lo aprendido.

Sin embargo, en un estudio realizado recientemente en nuestro país los resultados indican que los estilos de aprendizaje de los estudiantes de enfermería no se modifican en las diferentes etapas de su formación ${ }^{14}$.

Es evidente, que los datos de nuestro estudio no nos permiten afirmar que los alumnos modifican su estilo de aprendizaje a lo largo de su formación, determinando un perfil específico de la disciplina. Pensamos que para obtener una mayor evidencia científica sería necesario llevar a cabo un estudio longitudinal desde primero hasta tercer curso y ampliar el ámbito de estudio a otros centros cuya orientación metodológica sea diferente, con el fin de analizar también si existe relación entre estilo de enseñanza y estilo de aprendizaje.

Como docentes nos planteamos que con nuestro estilo de enseñar y las estrategias didácticas que utilizamos, posiblemente estemos encauzando las pre- ferencias de los alumnos en un sentido. Si el estudio de la disciplina de enfermería requiere un perfil teórico-reflexivo o los docentes lo estamos potenciando con los métodos utilizados, cabe esperar que los estudiantes con mayor preferencia por estos estilos tendrán un mejor rendimiento académico a lo largo de su formación, mientras que los alumnos con un perfil más activo-pragmático tendrán más dificultades y, por lo tanto, necesitarán un mayor apoyo por parte del profesorado. Es necesario buscar alternativas para potenciar el desarrollo de estilos activo y pragmático en la misma medida que los estilos reflexivo y teórico, ya que así el alumno va a tener más facilidad para aprender de cualquier forma y aprovechar las oportunidades de aprendizaje que se encuentre a lo largo de su vida.

\section{AGRADECIMIENTOS}

Agradecemos a Catalina Alonso García y a Domingo Gallego Gil, profesores del Departamento de Didáctica de la Facultad de Educación de la UNED, sus orientaciones en el diseño de este estudio.

\section{BIBLIOGRAFÍA}

1. García E, Pascual F. Estilos de aprendizaje y cognitivos. En: Puente A. Estilos de aprendizaje y enseñanza. Madrid: CEPE, S.A.; 1994. p 30.

2. Alonso C. Estilos de aprendizaje y estudiantes universitarios. En Gallego D, Alonso C. Estilos de Aprender y Estilos de Enseñar. Curso de Doctorado: UNED; 20012002. p. 39.

3. Alonso C, Gallego D, Honey P. Los estilos de aprendizaje. Procedimientos de diagnóstico y mejora. Bilbao: Mensajero; 1994. p. 55.

4. Hernando J. El aprendizaje individual permanente: ¿Cómo lograr el desarrollo de esta capacidad de los estudiantes?. Cartilla docente, publicaciones del CREA Universidad ICESI. < http://www.icesi.edu.co >

5. Alonso C. Estilos de aprendizaje, tutorías y enseñanza a distancia. En Gallego D, Alonso C. Estilos de Aprender y Estilos de Enseñar. Curso de Doctorado: UNED; 2001-2002. p. 23.

6. Alonso C, Gallego D, Honey P. Los estilos de aprendizaje. Procedimientos de diagnóstico y mejora. Bilbao: Mensajero; 1994. p. 70.

7. Alonso C, Gallego D, Honey P. Los estilos de aprendizaje. Procedimientos de diagnóstico y mejora. Bilbao: Mensajero; 1994. p. 71-74.

8. Highfield M E. Learning Styles. Nurse Educator. 1988; 13 (6): $30-33$. 
9. Rakoczy M, Money S. Learning styles of nursing students: a 3-year cohort longitudinal study. Journal of Professional Nursing. 1995; 11 (3):170-174.

10. Cavanagh SJ, Hogan K, Ramgopal T. The assessment of student nurse learning styles using the Kolb Learning Styles Inventori. Nurse Education Today. 1995; 15 (3): 177-183.

11. Linares AZ. Learning styles of students and faculty in selected health care professions. Journal of Nursing Education. 1999; 38 (9): 407-414.

12. Alonso C. Estilos individuales de aprendizaje: implicaciones en la conducta vocacional. En Gallego D, Alonso C. Estilos de aprender y estilos de enseñar. Curso de Doctorado: UNED; 2001-2002. P.107.
13. Alonso C. Análisis y diagnóstico de los estilos de aprendizaje en estudiantes universitarios. Madrid: Universidad Complutense; 1992.

14. Alonso C, Gallego D y Honey P. Cuestionario HoneyAlonso de Estilos de aprendizaje CHAEA. En: www.aprender.org.ar/aulas/avadim/recursos/ CHAEA1.rtf

15. Ordoñez FJ, Rosety-Rodriguez M, Rosety-Plaza M. Análisis de los estilos de aprendizaje predominantes entre los estudiantes de ciencias de la salud. Enfermeria global. 2003;(3). <http://www.um.es/eglobal/3/03c04.html

\section{ANEXO: CUESTIONARIO HONEY-ALONSO DE ESTILOS DE APRENDIZAJE; CHAEA C. M. ALONSO, D. J. GALLEGO Y P. HONEY}

\section{Instrucciones para responder al cuestionario}

- Este cuestionario ha sido diseñado para identificar su Estilo preferido de Aprendizaje. No es un test de inteligencia, ni de personalidad

- No hay límite de tiempo para contestar al Cuestionario. No le ocupará más de 15 minutos.

- No hay respuestas correctas o erróneas. Será útil en la medida que sea sincero/a en sus respuestas.

- Si está más de acuerdo que en desacuerdo con el ítem seleccione 'Mas (+)'. Si, por el contrario, está más en desacuerdo que de acuerdo, seleccione 'Menos (-)'.

- Por favor conteste a todos los items.

- El Cuestionario es anónimo. Para facilitar el análisis del grupo le rogamos que responda también a las preguntas de índole socioacadémica.

Muchas gracias.

1. Tengo fama de decir lo que pienso claramente y sin rodeos.

2. Estoy seguro de lo que es bueno y lo que es malo, lo que esta bien y lo que esta mal.

3. Muchas veces actúo sin mirar las consecuencias.

4. Normalmente trato de resolver los problemas metódicamente y paso a paso.

5. Creo que los formalismos coartan y limitan la actuación libre de las personas.

6. Me interesa saber cuales son los sistemas de valores de los demás y con qué criterios actúan.

7. Pienso que el actuar intuitivamente puede ser siempre tan valido como actuar reflexivamente.

8. Creo que lo más importante es que las cosas funcionen.

9. Procuro estar al tanto de lo que ocurre aquí y ahora.

10. Disfruto cuando tengo tiempo para preparar mi trabajo y realizarlo a conciencia.

11. Estoy a gusto siguiendo un orden, en las comidas, en el estudio, haciendo ejercicio regularmente.
12. Cuando escucho una nueva idea enseguida comienzo a pensar como ponerla en práctica.

13. Prefiero las ideas originales y novedosas aunque no sean prácticas.

14. Admito y me ajusto a las normas solo si me sirven para lograr mis objetivos.

15. Normalmente encajo bien con personas reflexivas, y me cuesta sintonizar con personas demasiado espontáneas, imprevisibles.

16. Escucho con más frecuencia que hablo.

17. Prefiero las cosas estructuradas a las desordenadas.

18. Cuando poseo cualquier información, trato de interpretarla bien antes de manifestar alguna conclusión.

19. Antes de hacer algo estudio con cuidado sus ventajas e inconvenientes.

20. Crezco con el reto de hacer algo nuevo y diferente.

21. Casi siempre procuro ser coherente con mis criterios y sistemas de valores. Tengo principios y los sigo.

22. Cuando hay una discusión no me gusta ir con rodeos. 
23. Me disgusta implicarme afectivamente en mi ambiente de trabajo. Prefiero mantener relaciones distantes.

24. Me gustan más las personas realistas y concretas que las teóricas.

25. Me gusta ser creativo, romper estructuras.

26. Me siento a gusto con personas espontáneas y divertidas.

27 La mayoría de las veces expreso abiertamente cómo me siento.

28. Me gusta analizar y dar vueltas a las cosas.

29. Me molesta que la gente no se tome en serio las cosas.

30. Me atrae experimentar y practicar las últimas técnicas ç y novedades.

31. Soy cauteloso a la hora de sacar conclusiones.

32. Prefiero contar con el mayor número de fuentes de información. Cuantos más datos reúna para reflexionar, mejor.

33. Tiendo a ser perfeccionista.

34. Prefiero oír las opiniones de los demás antes de exponer la mía.

35. Me gusta afrontar la vida espontáneamente y no tener que planificar todo previamente.

36. En las discusiones me gusta observar cómo actúan los demás participantes.

37. Me siento incómodo con las personas calladas y demasiado analíticas.

38. Juzgo con frecuencia las ideas de los demás por su valor práctico.

39. Me agobio si me obligan a acelerar mucho el trabajo para cumplir un plazo.

40. En las reuniones apoyo las ideas prácticas y realistas.

41. Es mejor gozar del momento presente que deleitarse pensando en el pasado o en el futuro.

42. Me molestan las personas que siempre desean apresurar las cosas.

43. Aporto ideas nuevas y espontáneas en los grupos de discusión.

44. Pienso que son más conscientes las decisiones fundamentadas en un minucioso análisis que las basadas en la intuición.

45. Detecto frecuentemente la inconsistencia y puntos débiles en las argumentaciones de los demás.

46. Creo que es preciso saltarse las normas muchas más veces que cumplirlas.

47. A menudo caigo en cuenta de otras formas mejores y más prácticas de hacer las cosas.

48. En conjunto hablo más que escucho.

49. Prefiero distanciarme de los hechos y observarlos desde otras perspectivas.

50. Estoy convencido que deber imponerse la lógica y el razonamiento.

51. Me gusta buscar nuevas experiencias.
52. Me gusta experimentar y aplicar las cosas.

53. Pienso que debemos llegar pronto al grano, al meollo de los temas.

54. Siempre trato de conseguir conclusiones e ideas claras.

55. Prefiero discutir cuestiones concretas y no perder el tiempo con charlas vacías.

56. Me impaciento cuando me dan explicaciones irrelevantes e incoherentes.

57. Compruebo antes si las cosas funcionan realmente.

58. Hago varios borradores antes de la redacción definitiva de un trabajo.

59. Soy consciente de que en las discusiones ayudo a mantener a los demás centrados en el tema, evitando divagaciones.

60. Observo que, con frecuencia, soy uno de los más objetivos y desapasionados en las discusiones.

61. Cuando algo va mal le quito importancia y trato de hacerlo mejor.

62. Rechazo ideas originales y espontáneas si no las veo prácticas.

63. Me gusta sopesar diversas alternativas antes de tomar una decisión.

64. Con frecuencia miro hacia delante para prever el futuro.

65. En los debates y discusiones prefiero desempeñar un papel secundario antes que ser el/la líder o el/la que más participa.

66. Me molestan las personas que no actúan con lógica.

67. Me resulta incomodo tener que planificar y prever las cosas.

68. Creo que el fin justifica los medios en muchos casos.

69. Suelo reflexionar sobre los asuntos y problemas.

70. El trabajar a conciencia me llena de satisfacción y orgullo.

71. Ante los acontecimientos trato de descubrir los principios y teorías en que se basan.

72. Con tal de conseguir el objetivo que pretendo soy capaz de herir sentimientos ajenos.

73. No me importa hacer todo lo necesario para que sea efectivo mi trabajo.

74. Con frecuencia soy una de las personas que más anima las fiestas.

75. Me aburro enseguida con el trabajo metódico y minucioso.

76. La gente con frecuencia cree que soy poco sensible a sus sentimientos.

77. Suelo dejarme llevar por mis intuiciones.

78. Si trabajo en grupo procuro que se siga un método y un orden.

79. Con frecuencia me interesa averiguar lo que piensa la gente.

80. Esquivo los temas subjetivos, ambiguos y poco claros. 


\section{PERFIL DE APRENDIZAJE}

1.- Rodee con una línea cada uno de los números que ha señalado con un signo más (+)

2.- Sume el número de círculos que hay en cada columna.

3.- Coloque estos totales en la gráfica. Así comprobará cual es su estilo o estilos de aprendizaje preferentes.

$\begin{array}{llll}\text { I } & \text { II } & \text { III } & \text { IV } \\ 3 & 10 & 2 & \text { I } \\ 5 & 16 & 4 & 8 \\ 7 & 18 & 6 & 12 \\ 9 & 19 & 11 & 14 \\ 13 & 28 & 15 & 22 \\ 20 & 31 & 17 & 24 \\ 26 & 32 & 21 & 30 \\ 27 & 34 & 23 & 38 \\ 35 & 36 & 25 & 40 \\ 37 & 39 & 29 & 47 \\ 41 & 42 & 33 & 52 \\ 43 & 44 & 45 & 53 \\ 46 & 49 & 50 & 56 \\ 48 & 55 & 54 & 57 \\ 51 & 58 & 60 & 59 \\ 61 & 63 & 64 & 62 \\ 67 & 65 & 66 & 68 \\ 74 & 69 & 71 & 72 \\ 75 & 70 & 78 & 73 \\ 77 & 79 & 80 & 76\end{array}$

Totales:

Grupo

Activo

Reflexivo

Teórico

Pragmático

GRÁFICA DE ESTILOS DE APRENDIZAJE

Dibuja los cuatro puntos de los totales en la gráfica y únelos con una línea recta.

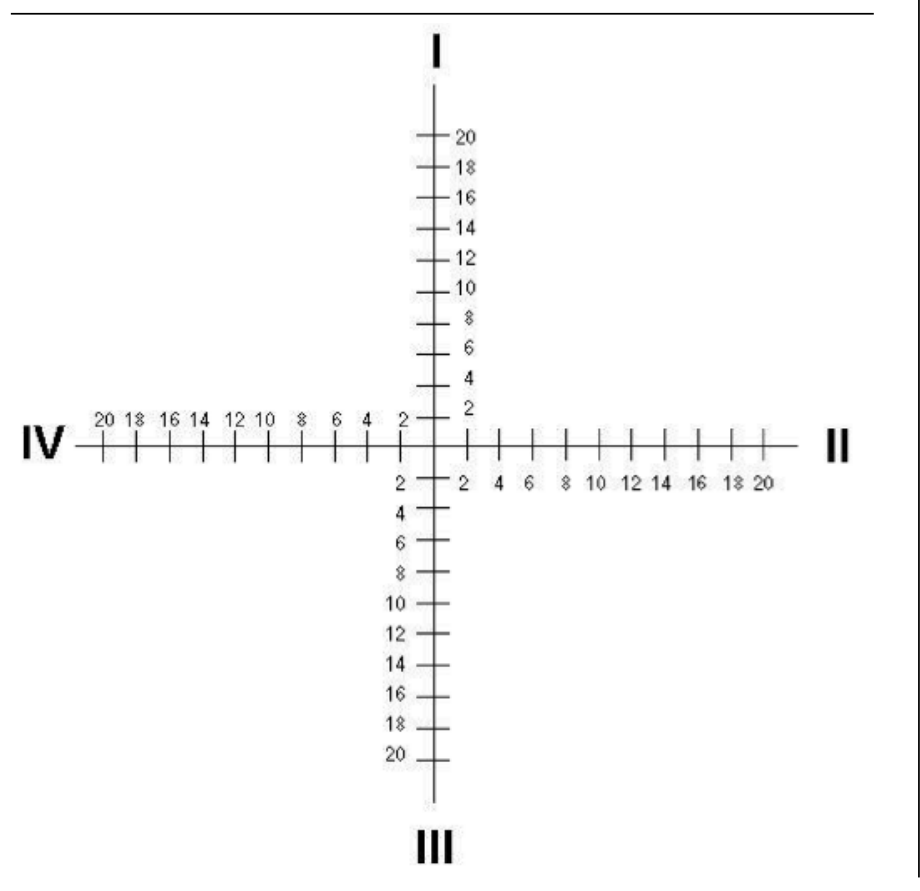

FICHA DE DATOS

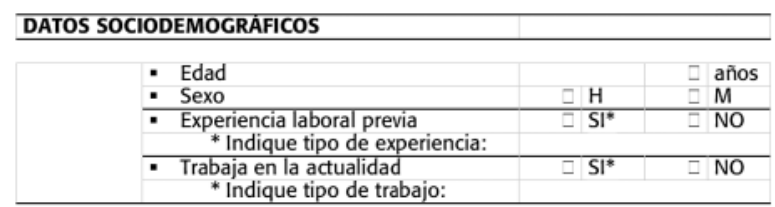

DATOS ACADÉMICOS

- Curso

Acceso a los estudios de Enfermería

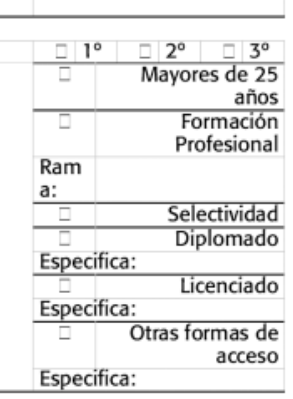

